7-8-2011

\title{
Direct Patterning of a Cyclotriveratrylene Derivative for Directed Self-Assembly of C60
}

\author{
Zachary R. Osner \\ Loyola University Chicago \\ Dorjderem Nyamjav \\ Loyola University Chicago \\ Richard C. Holz \\ Loyola University Chicago \\ Daniel P. Becker \\ Loyola University Chicago, dbecke3@luc.edu
}

Follow this and additional works at: https://ecommons.luc.edu/chemistry_facpubs

Part of the Chemistry Commons

\section{Author Manuscript}

This is a pre-publication author manuscript of the final, published article.

\section{Recommended Citation}

Osner, Zachary R.; Nyamjav, Dorjderem; Holz, Richard C.; and Becker, Daniel P.. Direct Patterning of a Cyclotriveratrylene Derivative for Directed Self-Assembly of C60. Nanotechnology, 22, 27: , 2011. Retrieved from Loyola eCommons, Chemistry: Faculty Publications and Other Works, http://dx.doi.org/10.1088/ 0957-4484/22/27/275611

This Article is brought to you for free and open access by the Faculty Publications and Other Works by Department at Loyola eCommons. It has been accepted for inclusion in Chemistry: Faculty Publications and Other Works by an authorized administrator of Loyola eCommons. For more information, please contact ecommons@luc.edu. (c) (i) $\odot$

This work is licensed under a Creative Commons Attribution-Noncommercial-No Derivative Works 3.0 License. (c) 2011 Institute of Physics 


\section{Direct Patterning of a Cyclotriveratrylene (CTV) Derivative for Directed Self-Assembly of $\mathbf{C}_{60}$}

Zachary R. Osner, Dorjderem Nyamjav, Richard C. Holz*, and Daniel P. Becker*

Department of Chemistry, Loyola University Chicago, Chicago, Illinois 60626 
Keywords: $\mathrm{C}_{60}$, Cyclotriveratrylene, Supramolecular Chemistry, Guest-Host chemistry, DipPen Nanolithography, AFM, TMAFM.

*Address correspondence to: Daniel Becker, Department of Chemistry, Loyola University Chicago, 1068 W. Sheridan Rd., Chicago IL 60626, Phone 773-580-3100, Fax: 773-797-3086, Internet: $\underline{\text { dbecke3@luc.edu. }}$

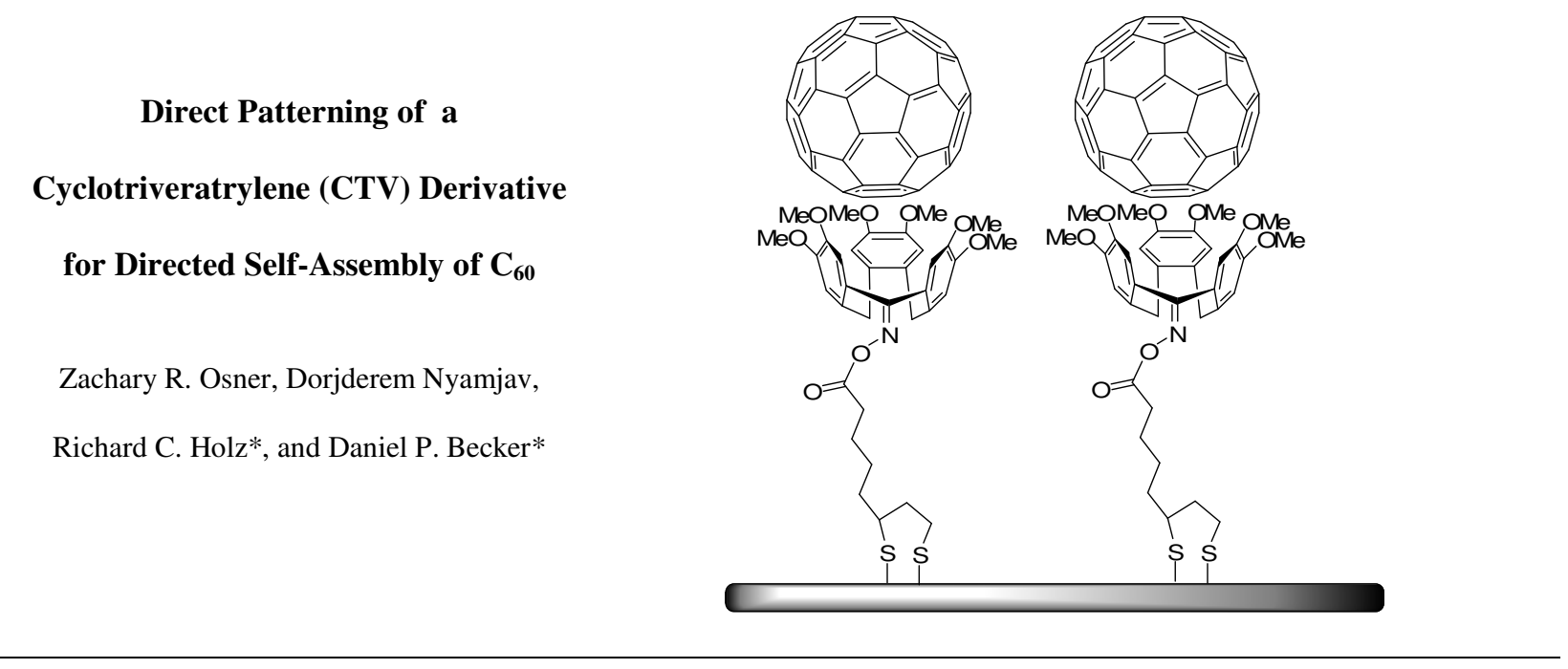




\begin{abstract}
A novel apex-modified cyclotriveratrylene (CTV) derivative with an attached thiolanecontaining lipoic acid linker was directly patterned onto gold substrates via Dip-Pen nanolithography (DPN). The addition of a dithiolane-containing linker to the apex of CTV provides a molecule that can adhere to a gold surface with its bowl shaped cavity directed away from the surface thereby providing a surface-bound CTV host that can be used for the directed assembly of guest molecules. Subsequent exposure of these CTV microarrays to $\mathrm{C}_{60}$ in toluene resulted in the directed assembly of predesigned, spatially controlled, high-density microarrays of $\mathrm{C}_{60}$. The molecular recognition capabilities of this $\mathrm{CTV}$-template toward $\mathrm{C}_{60}$ provides proofof-concept that supramolecular CTV scaffolds can be directly patterned onto surfaces providing a foundation for the development of organic electronic and optoelectronic materials.
\end{abstract}


With its unique structure, physical, and electronic properties, $\mathrm{C}_{60}$ (Buckminsterfullerene) has been shown to possess great potential for the development of organic electrical and optical devices. $^{1-4}$ For example, $\mathrm{C}_{60}$ is a good electrical conductor at the nanoscale, nearly as good as copper metal. $\mathrm{C}_{60}$ is also a good thermal conductor and is one of the strongest materials known, being 100 times stronger than steel but one-sixth the weight. ${ }^{5-7}$ Moreover, the ability of $\mathrm{C}_{60}$ to be a potent electron acceptor has led to its utilization in donor-chromophore-acceptor based molecular triads that are capable of intramolecular photoinduced electron transfer (PET). ${ }^{8}$ While $\mathrm{C}_{60}$ thin-films on metal surfaces have been widely studied, ${ }^{3,9-14}$ many challenges remain for the directed self-assembly of organic optoelectronic materials such as $\mathrm{C}_{60}$ into two-dimensional surface structures. Therefore, developing methods to pattern and immobilize organic electronic or optoelectronic materials with nanometer-scale control will provide a simple, robust, and flexible approach for the preparation of predetermined two-dimensional organic materials. By controlling the spatial distribution of organic molecules on a surface by directed molecular binding, these materials will potentially allow for the development of new nanooptical, nanoelectronic, and/or nanoelectrochemical systems (NEMS)., ${ }^{3,-12}$

One way to pattern and immobilize organic electronic or optoelectronic materials with nanometer scale control is to utilize a bottom-up, layer-by-layer approach based on host-guest chemistry. ${ }^{3}$ Host-guest chemistry involves complementary binding between two different molecules that can involve electrostatic, hydrogen bonding, $\pi-\pi$ stacking interactions, inductive and dispersion forces, as well as hydrophobic or solvatophobic effects. ${ }^{15}$ Over the past decade, host-guest chemistry involving synthetic receptor molecules has received increasing interest partly due to the ever-advancing ability to synthesize complex molecular scaffolds to serve as host structures. One such receptor, cyclotriveratrylene (CTV), ${ }^{16-18}$ has been extensively employed in host-guest chemistry as a supramolecular scaffold. ${ }^{19-21}$ Enabled by its rigid bowl- 
shaped structure, CTV has been shown to act as a host molecule for a variety of small molecules including neutral or ionic polyhedral $\mathrm{C}_{60}$ and $o$-carborane derivatives. ${ }^{22,23}$ In 1994, Atwood $e t$ $a l .^{24}$ showed that the bowl-shaped crown conformer of CTV forms inclusion complexes with $\mathrm{C}_{60}$ in the ratio of $\left(\mathrm{C}_{60}\right)_{1.5}(\mathrm{CTV})$ (toluene $)_{0.5}$ referred to as a "ball and socket" structure. Zhang et $a l .{ }^{25-28}$ utilized this ball and socket structure to prepare $\mathrm{C}_{60}$ self-assembled monolayers (SAMs) on gold utilizing CTV, however the CTV was derivatized on its perimeter resulting in the concave shape of the CTV molecule facing toward the gold surface, thus irreversibly trapping $\mathrm{C}_{60}$ against the surface and isolating it from neighboring CTV guests. This orientation of CTV prohibits its ability to function as a template for a layer-by-layer approach to building organic electronic or optoelectronic materials.

Herein we describe a robust and reliable method to produce predesigned, spatially controlled, high-density microarrays of $\mathrm{C}_{60}$. We have designed and synthesized an apexmodified CTV derivative providing a surface bound CTV template with its bowl shaped cavity directed away from the surface. By utilizing a layer-by-layer approach and Dip-Pen Nanolithography (DPN), which provides a flexible nanolithographic method capable of positioning molecules on a substrate with $10 \mathrm{~nm}$ resolution, ${ }^{29,30}$ predesigned, spatially controlled microarrays of this modified CTV derivative were prepared on gold surfaces. The molecular recognition capabilities of this CTV-template towards $\mathrm{C}_{60}$ provides proof-of-concept that supramolecular CTV scaffolds can be directly patterned on surfaces and through host-guest interations provide a template for the development of organic electronic or optoelectronic materials.

\section{Materials and Methods}

Materials. All solvents and reagents used were purchased from Sigma-Aldrich 
(Milwaukee, WI) and were used as received without further purification.

Synthesis of 10,15-Dihydro-2,3,7,8,12,13-hexamethoxy-5H-tribenzo[a,d,g]-cyclononenO-[5-(1,2-dithiolan-3-yl)pentanoyl]-5-oxime (2a/b). CTV ketone and CTV oxime were prepared by a modification of the previously published procedure. ${ }^{31}$ For the synthesis of the crown and saddle CTV oxime-lipoic acid derivatives, lipoic acid (283 mg, $1.37 \mathrm{mmol})$, hydroxybenzotriazole (HOBT) (200 mg, 1.48 mmol), N,N'-dicyclohexylcarbodiimide (DCC) (330 mg, $1.60 \mathrm{mmol}$ ), and $3.0 \mathrm{~mL}$ of dry tetrahydrofuran (THF) were mixed in a round bottom flask and was allowed to stir at room temperature for 1 hour. A mixture of crown and saddle conformers of CTV oxime (545 mg, $1.14 \mathrm{mmol}$ ) in $2.7 \mathrm{~mL}$ of dry THF was then added to the round bottom flask dropwise. The reaction was allowed to stir for $\sim 24$ hours and was monitored via TLC using ethyl acetate/dichloromethane (EA/DCM, 1/9) as the eluent. Upon consumption of the starting material, the reaction was filtered over celite to remove the insoluble urea and washed with $\sim 30 \mathrm{~mL}$ of DCM. The solvent was then removed under reduced pressure. The crude mixture was purified using flash chromatography with an RS-40 cartridge and an eluent gradient of EA/hexane (1/2 to $2 / 1, \mathrm{v} / \mathrm{v})$. The crown/saddle (2a/b) fractions were combined and removal of solvent afforded a light brown solid was recovered (434 mg, yield: 57\%). This product was characterized by ${ }^{1} \mathrm{H}$ NMR, ${ }^{13} \mathrm{C}$ NMR, and IR. ${ }^{1} \mathrm{H}$ NMR $\left(300 \mathrm{MHz}, \mathrm{CDCl}_{3}\right.$, TMS as internal standard) $\delta 7.33(\mathrm{~s}, 1 \mathrm{H}), 6.94(\mathrm{~s}, 1 \mathrm{H}), 6.93(\mathrm{~s}, 1 \mathrm{H}), 6.91(\mathrm{~s}, 1 \mathrm{H}), 6.83(\mathrm{~s}, 1 \mathrm{H}), 6.80(\mathrm{~s}$, 1H), $6.78(\mathrm{~s}, 1 \mathrm{H}), 6.68(\mathrm{~s}, 1 \mathrm{H}), 6.65(\mathrm{~s}, 1 \mathrm{H}), 6.64(\mathrm{~s}, 1 \mathrm{H}), 6.58(\mathrm{~s}, 1 \mathrm{H}), 6.54(\mathrm{~s}, 1 \mathrm{H})$.

Preparation of Gold Substrates. Silicon oxide substrates with $500 \mathrm{~nm}$ thermally evaporated oxide layers were purchased from WaferNet, Inc. (CA). Thin films of $\mathrm{Cr}$ and $\mathrm{Au}$ with thicknesses of $10 \mathrm{~nm}$ and $30 \mathrm{~nm}$, respectively, were evaporated onto pre-cut, piranha (3:1 = $\mathrm{H}_{2} \mathrm{SO}_{4}: \mathrm{H}_{2} \mathrm{O}_{2}$ ) cleaned silicon pieces using an Edwards Auto 306 system. (Caution! Piranha solution should be handled carefully as it may cause serious burns.) 
Fabrication of CTV Microarrays. A NanoInk, Inc. Nscriptor ${ }^{\mathrm{TM}}$ was used to prepare DPN arrays under ambient conditions with temperatures ranging from 20 to $22^{\circ} \mathrm{C}$ and humidity levels within the enclosed chamber between 25 and 35\%. V-shaped, silicon nitride contact mode tips (NanoInk, Inc.) with a spring constant of $0.5 \mathrm{~N} / \mathrm{m}$ were used for DPN patterning. A $10 \mathrm{mM}$ solution of $\mathbf{2} \mathbf{a} / \mathbf{b}$ was prepared in acetonitrile containing $1 \%$ polysorbate 20 for wettability. For DPN patterning, atomic force microscopy (AFM) tips were first dipped into inkwells filled with $\mathbf{2 a} / \mathbf{b}$. The stationary diffusion constants were calculated based on the model developed by J. Jang et al. ${ }^{32}$ prior to each patterning process. Dot shaped patterns were made by holding the tip stationary in contact with the surface. The samples with DPN arrays were allowed to stand at room temperature for $\sim 10$ minutes and rinsed with acetonitrile then ethanol and dried under a stream of nitrogen.

Modification of the DPN-patterned templates. Substrates with DPN-generated patterns were incubated in a $1 \mathrm{mM}$ solution of octadecanethiol (ODT) in ethanol for $\sim 30$ min to block any exposed gold surface from further unwanted contaminations or modifications. The samples were then rinsed with ethanol and dried under a stream of nitrogen. Regions coated with $\mathbf{2 a} \mathbf{a} / \mathbf{b}$ were then functionalized using a $1 \mathrm{mM}$ solution of $\mathrm{C}_{60}$ in toluene with deposition times ranging from 20 minutes to 1 hour. Functionalized samples were rinsed with toluene and dried under a stream of nitrogen.

Imaging and Surface Characterization. Fabricated microarrays were characterized by AFM. A NanoInk, Inc. Nscriptor ${ }^{\mathrm{TM}}$ was employed to acquire topography, phase and frictional force images. A v-shaped, silicon nitride contact mode cantilever tip (model MSCT-AUNM-10 purchased from Veeco, Inc) with a spring constant of $0.05 \mathrm{~N} / \mathrm{m}$ was used for lateral force microscopy (LFM) images while a beam shaped, silicon tapping mode tip with a spring constant 
of 40 N/m, from Pacific Nanotechnology, was used for Tapping Mode AFM (TMAFM) imaging. All the AFM images were acquired with resolutions of 512 x 512 pixels. Self-assembled monolayers for matrix assisted laser desorption ionization mass spectrometry (SAMDI-TOF MS) spectra were obtained using a 4800 MALDI-TOF/TOF (Applied Biosystems, Farmingham, MA) with a $335 \mathrm{~nm} \mathrm{Nd:YAG} \mathrm{laser} \mathrm{as} \mathrm{a} \mathrm{desorption/ionization} \mathrm{source} \mathrm{using} \mathrm{a} \mathrm{matrix} \mathrm{of} \mathrm{2,4,6-}$ trihydroxyacetophenone, $25 \mathrm{mg} / \mathrm{mL}$ in acetonitrile. All spectra were acquired with $20 \mathrm{kV}$ accelerating voltage using positive reflector mode. The extraction delay was $450 \mathrm{~ns}, 1200$ laser shots were applied, and the entire surface of the circle was sampled. Each spectrum was calibrated using the EG3-EG3 disulfide background as an internal standard.

\section{Results and Discussion}

We hypothesized that derivatizing the apex of the CTV bowl would provide a supramolecular scaffold with the concave bowl receptor pointed away from the surface, enabling CTV to function as a surface-bound host molecule. To accomplish this, CTV was oxidized to the monoketone and converted to the oxime in high yield as an equilibrium mixture of the crown $\mathbf{1 a}$ and the saddle $\mathbf{1 b}$ conformers (Figure 1). ${ }^{16}$ The CTV oxime was coupled to ( \pm )- $\alpha$-lipoic acid affording a mixture of the coupled crown (2a) and saddle (2b) conformers in $52 \%$ yield (Figure 1). The resulting CTV-lipoic acid derivatives $(\mathbf{2 a} / \mathbf{b})$ contain a dithiolane-terminated linker for coordination to gold, thus enabling the bowl of CTV to face away from the surface.

With the successful design and synthesis of an apex modified CTV supramolecular scaffold head group with a dithiolane tail, microarrays of $\mathbf{2 a} / \mathbf{b}$ were prepared via DPN by direct patterning using a NanoInk, Inc. Nscriptor ${ }^{\mathrm{TM}}$ system. $^{29,30}$ DPN is a particularly important 
nanolithographic method for patterning molecular inks since DPN is capable of positioning molecules on a substrate with $10 \mathrm{~nm}$ resolution in pre-designed, spatially controlled arrays. ${ }^{29}$ As previously reported, the addition of $1 \%$ polysorbate 20 to a $10 \mathrm{mM}$ solution of $\mathbf{2 a} / \mathbf{b}$ in acetonitrile enhanced the diffusion of $\mathbf{2} \mathbf{a} / \mathbf{b}$ during the DPN process by making the solvent more wettable. ${ }^{33}$ DPN generated patterns of $\mathbf{2 a} / \mathbf{b}$ were prepared at $20^{\circ} \mathrm{C}$ with humidity levels between 25 and $35 \% .^{34}$ To assess the adsorption of the $\mathbf{2 a} / \mathbf{b}$ molecular ink, surface topography changes were measured by AFM (LFM and TMAFM) after curing in air at room temperature for $\sim 10$ minutes, followed by rinsing with acetonitrile and ethanol and drying under a stream of nitrogen. LFM is a method of imaging a surface by detecting the change in the torsion of the cantilever as the cantilever tip encounters a change in friction on the surface as the tip moves in the forward and reverse direction. Typical AFM images of DPN-generated $\mathbf{2 a} / \mathbf{b}$ patterns are shown in Figure 2. Surface-bound $\mathbf{2 a} / \mathbf{b}$, which is more hydrophilic than gold, is observed as the light contrast areas in the TMAFM image (Figure 2b) and a darker contrast in the LFM image (Figures 2b).

The estimated height of DPN generated patterns of $\mathbf{2 a} / \mathbf{b}$, measured from randomly placed height profiles using tapping-mode AFM revealed a height of $1.2 \pm 0.3 \mathrm{~nm}$ every $1.5 \mu \mathrm{m}$ with a width of $0.5 \mu \mathrm{m}$ (Figure 2c). The calculated height of a $\mathbf{2 a} / \mathbf{b}$ monolayer is $1.5 \mathrm{~nm}$, consistent with the experimentally observed height values obtained for $\mathbf{2 a} / \mathbf{b}$, indicating the formation of a 2a/b SAM. Gold substrates containing $\mathbf{2 a} / \mathbf{b}$ SAMs were immersed in a $1 \mathrm{mM}$ solution of ODT in ethanol for $~ 30 \mathrm{~min}$, rinsed with ethanol, and dried under a stream of nitrogen. A tappingmode AFM topography image after passivation with ODT and a corresponding height profile are shown in Figure 3a. Tapping-mode AFM images demonstrate the comparable heights of spots containing the $\mathbf{2 a} / \mathbf{b}$ SAM and the surrounding ODT back-filled resist layers due to the similar heights of their SAMs (ca. $1.5 \mathrm{~nm}$ vs. $1.8 \mathrm{~nm}$, respectively). ${ }^{35}$ However, microarrays of $\mathbf{2 a / b}$ backfilled with ODT can be clearly differentiated by LFM due to the greater frictional force and 
darker contrast in comparison to the ODT resist layer between the AFM tip and the $\mathbf{2 a / b}$ SAM (Figure 3b). The graininess of the images shown in Figures $3 a$ and $3 b$ reveal the granularity of the gold surface, which was not investigated further.

The molecular recognition capabilities of the DPN generated CTV-template microarrays towards $\mathrm{C}_{60}$ were examined. Arrays of $\mathbf{2 a} / \mathbf{b}-\mathrm{ODT}$ were immersed in a $1 \mathrm{mM}$ solution of $\mathrm{C}_{60}$ in toluene for $\sim 40$ min and after extensive rinsing with toluene they were dried with nitrogen and characterized via AFM. A typical AFM image showing $\mathrm{C}_{60}$ attached to the CTV-template is presented in Figure 4a. The binding interaction of the $\mathbf{2 a} / \mathbf{b}-$ ODT SAMs with $\mathrm{C}_{60}$ results in a light TMAFM contrast (Figure 4a) and a clearly visible contrast in the LFM image (Figure 4b). This is in good agreement with the frictional behavior of $\mathrm{C}_{60}$, which is somewhat more hydrophilic than ODT. $\mathrm{C}_{60}$ was observed bound to individual dots with little or no binding to the resist ODT monolayer. Additional confirmation of $\mathrm{C}_{60}$ binding to DPN generated $\mathbf{2 a} / \mathbf{b}$ templates was obtained from SAMDI-TOF mass spectroscopy. Mrksich and co-workers have shown that SAMDI-TOF MS is an excellent tool to directly detect organic molecules, such as synthetic intermediates, at surfaces. ${ }^{36}$ Therefore, SAMDI-TOF MS spectra were collected on DPN generated SAMS of $\mathbf{2} \mathbf{a} / \mathbf{b}$ before and after exposure to $\mathrm{C}_{60}$. For SAMs of $\mathbf{2 a} / \mathbf{b}$, a significant (74\%) $\mathrm{m} / \mathrm{z}$ peak at 462.27 was observed, which we assign to the cleavage product of $\mathbf{2} \mathbf{a} / \mathbf{b}$ at the N-O bond (Figure 1). No parent peak was observed for $\mathbf{2} \mathbf{a} / \mathbf{b}$ and no peaks at larger masses were detected indicating that the lipoic acid tail of $\mathbf{2} \mathbf{a} / \mathbf{b}$ was lost in the laser desorption process. Exposure of DPN generated SAMS of $\mathbf{2 a} / \mathbf{b}$ to $\mathbf{C}_{60}$ resulted in the observation of an $\mathrm{m} / \mathrm{z}$ peak at 720.05 in the SAMDI-TOF mass spectrum indicating the presence of $\mathrm{C}_{60}$ bound to the $\mathbf{2 a} / \mathbf{b}$ monolayer. These data, taken together, provide proof-of-concept that supramolecular CTV 
scaffolds can be directly patterned on surfaces and retain their ability to bind host molecules such as $\mathrm{C}_{60}$.

Given that the diameter of $\mathrm{C}_{60}$ is $\sim 1 \mathrm{~nm}$, a height increase for a SAM of $\mathbf{2} \mathbf{a} / \mathbf{b}$ after the addition of $\mathrm{C}_{60}$ is expected if $\mathrm{C}_{60}$ binds to the $\mathrm{CTV}$ macrocycle in a ball and socket fashion as observed by Atwood et al. ${ }^{24}$ in the solid state. AFM height profiles of the $\mathbf{2} \mathbf{a} / \mathbf{b}-\mathrm{C}_{60} \mathrm{SAM}$ after the addition of the ODT resist was found to be $1.0 \pm 0.3 \mathrm{~nm}$ (Figure $4 \mathrm{c}$ ). Since $\mathbf{2} \mathbf{a} / \mathbf{b}$ and ODT have approximately the same height, the $1 \mathrm{~nm}$ height increase is evidence of $\mathrm{C}_{60}$ binding through $\pi-\pi$ interactions (Figure 5) similar to the binding mode reported for the solid state. ${ }^{24}$ Previously we had shown that apex-modified CTV derivatives interconvert between two different conformers, crown $\mathbf{1 a}$ and saddle $\mathbf{1 b}{ }^{31}$ The interconversion equilibrium between the two conformers was shown to be solvent dependent with the crown conformer being favored in nonpolar solvents. ${ }^{37}$ Given the nearly complete coverage of the CTV-surface bound template, the equilibrium between the crown conformer $\mathbf{2 a}$ and the saddle conformer $\mathbf{2} \mathbf{b}$ must be shifted towards 2a (Figure 1) enabling a ball-and-socket interaction between the host CTV molecules and the $\mathrm{C}_{60}$ guest. ${ }^{37}$ Therefore, the apex-bound lipoic acid-CTV molecule (2a) resides on the surface with its bowl shaped cavity directed away from the surface. The proposed conformation of the CTV bowl is consistent with other cyclophane SAMs, such as calix[n]arenes $(n=4,6$, $8)^{26,38}$

\section{Conclusion}

We have shown that an apex-modified CTV supramolecular scaffold can be patterned into pre-defined microarrays via DPN. Through host-guest interactions, these microarrays have been shown to form bottom-up, layer-by-layer complexes with $\mathrm{C}_{60}$ with potential towards 
advancing nanoelectronics and optoelectronics. Having the ability to directly pattern molecular host active surfaces via DPN opens the door to preparing a wide range of host-guest materials with reproducible, homogeneous features with high edge resolution, which will facilitate the fabrication of microcircuitry and opticalelectronics based on host-guest chemistry.

Acknowledgments. This work was supported by The Petroleum Research Fund (ACS PRF 50033-ND4, RCH). We would like to thank Professor Milan Mrksich and Dr. Adam Eisenberg at the University of Chicago for help in obtaining the SAMDI-TOF MS data. 


\section{References}

1. Mateo-Alonso, A.; Guldi, D. M.; Paolucci, F.; Prato, M., Fullerenes: Multitask

Components in Molecular Machinery. Angewandte Chemie International Edition 2007, 46, (43), 8120-8126.

2. Shirai, Y.; Guerrero, J. M.; Sasaki, T.; He, T.; Ding, H.; Vives, G.; Yu, B.; Cheng, L.; Flatt, A. K.; Taylor, P.; Gao, Y.; Tour, J. M., Fullerene/Thiol-Terminated Molecules. J. Org. Chem. 2009, 74.

3. Bonifazi, D.; Enger, O.; Diederich, F., Supramolecular [60]fullerene chemistry on surfaces. Chemical Society Reviews 2007, 36, (2), 390-414.

4. Song, J.; Aratani, N.; Shinokubo, H.; Osuka, A., A Porphyrin Nanobarrel That Encapsulates C60. Journal of the American Chemical Society 2010, 132, (46), 1635616357.

5. Appenzeller, J.; Joselevich, E.; Hoenlein, W., In Carbon Nanotubes for Data Processing; Nanoelectronics and Information Technology. Wiley-VCH: Weinheim: 2003; Vol. Vol. Chapter 19, p 1,002.

6. Avouris, P.; Appenzeller, J.; Martel, R.; Wind, S. J., Carbon Nanotube Electronics. Proc. IEEE 2003, 91, 1772-1784.

7. Dresselhaus, M. S.; Dresselhaus, G.; Avouris, P., Carbon Nanotubes: Synthesis, Structures, Properties, and Applications. In. Eds ed.; Springer-Verlag: Berlin, 2001; p 256.

8. Saha, S.; Johansson, E.; Flood, A. H.; Tseng, H.; Zink, J. I.; Stoddart, J. F., A Photoactive Molecular Triad as a Nanoscale Power Supply for a Supramolecular Machine. Chem. Eur. J. 2005, 11 . 
9. Chen, W.; Zhang, H.; Huang, H.; Chen, L.; Wee, A. T. S., Orientationally Ordered C60 on p-Sexiphenyl Nanostripes on $\operatorname{Ag}(111)$. ACS Nano 2008, 2, (4), 693-698.

10. Sedona, F.; Di Marino, M.; Sambi, M.; Carofiglio, T.; Lubian, E.; Casarin, M.; Tondello, E., Fullerene/Porphyrin Multicomponent Nanostructures on $\operatorname{Ag}(110)$ : From Supramolecular Self-Assembly to Extended Copolymers. ACS Nano 2010, 4, (9), 51475154.

11. Tait, S. L., Function Follows Form: Exploring Two-Dimensional Supramolecular Assembly at Surfaces. ACS Nano 2008, 2, (4), 617-621.

12. Zhao, J.; Feng, M.; Yang, J.; Petek, H., The Superatom States of Fullerenes and Their Hybridization into the Nearly Free Electron Bands of Fullerites. ACS Nano 2009, 3, (4), 853-864.

13. Zhong, D.; Wedeking, K.; Blo $\square$ mker, T.; Erker, G.; Fuchs, H.; Chi, L., Multilevel Supramolecular Architectures Self-Assembled on Metal Surfaces. ACS Nano 2010, 4, (4), 1997-2002.

14. Marois, J.-S. M., Jean-François, Synthesis and Surface Self-Assembly of [3]RotaxanePorphyrin Conjugates: Toward the Development of a Supramolecular Surface Tweezer for $\mathrm{C}_{60}$. Langmuir 2008, 24, 10865-10873.

15. Beer, P. D.; Gale, P. A.; Smith, D. K., Supramolecular Chemistry. Oxford University Press: Oxford, England, 1999.

16. Robinson, G. M., Reaction of Homopiperonyl and Homoveratryl Alcohols. J. Chem. Soc. $1915,107,267-276$.

17. Hardie, M. J. A., Ruksanna; Sumbly, Christopher J., Network structures of cyclotriveratrylene and its derivatives. New J. Chem. 2005, 29, 1231-1240. 
18. Brotin, T. D., Jean-Pierre, Cryptophanes and Their Complexes-Present and Future. Chem. Rev. 2009, 109, 88-130.

19. Huerta, E.; Isla, H.; Perez, E. M.; Bo, C.; Martin, N.; de Mendoza, J., Tripodal exTTF-CTV Hosts for Fullerenes. Journal of the American Chemical Society 2010, 132, (15), 53515353.

20. Hardie, M. J., Recent advances in the chemistry of cyclotriveratrylene. Chemical Society Reviews 2010, 39, (2), 516-527.

21. Carruthers, C.; Fisher, J.; Harding, L. P.; Hardie, M. J., Host-guest influence on metallosupramolecular assemblies with a cyclotriveratrylene-type ligand. Dalton Transactions 2010, 39, (2), 355-357.

22. Hardie, M. J. G., Peter D.; Raston, Colin L., Self-Assembly of Grid and Helical HydrogenBonded Arrays Incorporating Bowl-Shaped Receptor Sites that Bind Globular Molecules. Chem. Eur. J. 1999, 5, 1828-1833.

23. Ruksanna, A. F., Andreas; Kennedy, John D.; Hardie, Michael J., Group 1 Coordination Chains and Hexagonal Networks of Host Cyclotriveratrylene with Halogenated Monocarborane Anions. Chem. Eur. J. 2004, 10, 2190-2198.

24. Steed, J. W.; Junk, P. C.; Atwood, J. L.; Barnes, M. J.; Raston, C. L.; Burkhalter, R. S., Ball and Socket Nanostructures: New Supramolecular Chemistry Based on Cyclotriveratrylene. J. Am. Chem. Soc. 1994, 116, 10346-10347.

25. Zhang, S.; Palkar, A.; Fragoso, A.; Prados, P.; de Mendoza, J.; Echegoyen, L., Noncovalent Immobilization of C60 on Gold Surfaces by SAMs of Cyclotriveratrylene Derivatives. Chem. Mater. 2005, 17, 2063-2068. 
26. Zhang, S. E., L., Supramolecular Immobilization of Fullerenes on Gold Surfaces:

Receptors Based on Calix[n]Arenes, Cyclotriveratrylene (CTV) and Porphyrins. Comptes Rendus Chimie 2006, 9.

27. Nierengarten, J.; Oswald, L.; Eckert, J.; Nicoud, J.; Armaroli, N., Complexation of Fullerenes with Dendritic Cyclotriveratrylene Derivatives. Tetrahedron Lett. 1999, 40.

28. Nierengarten, J., Supramolecular Encapsulation of [60]Fullerene with Dendritic Cyclotriveratrylene Derivatives. Fullerenes, Nanotubes, and Carbon Nanostructures 2005, 13.

29. Piner, R. D.; Zhu, J.; Xu, F.; Hong, S.; Mirkin, C. A., "Dip-pen" nanolithography. Science 1999, 283, 661-663.

30. Salaita, K.; Wang, Y. H.; Fragala, J.; Vega, R. A.; Liu, C.; Mirkin, C. A., Massively parallel dip-pen nanolithography with 55000-pen two-dimensional arrays. Angewandte Chemie-International Edition 2006, 45, (43), 7220-7223.

31. Lutz Jr., M. R. F., D. C.; Rehage, P.; Becker, D. P. , Isolation of the Saddle and Crown Conformers of Cyclotriveratrylene (CTV) Oxime. Tetrahedron Lett. 2007, 48.

32. Jang, J.; Hong, S.; Schatz, G. C.; Ratner, M. A., Self-assembly of ink molecules in dip-pen nanolithography: A diffusion model. J. Chem. Phys. 2001, 115, 2711.

33. Nyamjav, D.; Holz, R. C., Direct Patterning of Silanized-Biomolecules on Semiconductor Surfaces. Langmuir 2010, null-null.

34. Jung, H.; Dalal, C. K.; Kuntz, S.; Shah, R.; Collier, C. P., Surfactant Activated Dip-Pen Nanolithography. Nano Lett. 2004, 4, (11), 2171-2177. 
35. Barczewski, M.; Walheim, S.; Heiler, T.; Bl $\square$ aszczyk, A.; Mayor, M.; Schimmel, T., High Aspect Ratio Constructive Nanolithography with a Photo-Dimerizable Molecule. Langmuir 2009, 26, (5), 3623-3628.

36. Ban, L. M., Milan, On-Chip Synthesis and Label-Free Assays of Oligosaccharide Arrays. Angew. Chem. Int. Ed. 2008, 47.

37. French, D. C.; Lutz, M. R., Jr.; Lu, C.; Zeller, M.; Becker, D. P., A Thermodynamic and Kinetic Characterization of the Solvent Dependence of the Saddle-Crown Equilibrium of Cyclotriveratrylene Oxime. J. Phys. Chem. A 2009, 113.

38. Zhang, S.; Echegoyen, L., Supramolecular Incorporation of Fullerenes on Gold Surfaces: Comparison of C60 Incorporation by Self-Assembled Monolayers of Different Calix[n]Arenes $(\mathrm{n}=4,6,8)$ Derivatives. J. Org. Chem. 2005, 70. 


\section{Figure Captions}

Figure 1. Synthetic scheme for the synthesis of the apex-modified dithiol CTV-oxime (1).

Figure 2. AFM generated images of $\mathbf{2 a} / \mathbf{b}$ dot patterns patterned onto the bare gold surface. a) TMAMF image showing height increase of $\mathbf{2 a / b}$ dot patterns patterned onto the bare gold surface via DPN. b) LFM images of $\mathbf{2} \mathbf{a} / \mathbf{b}$ patterned on a base gold substrate utilizing DPN. c) Step height profile from AFM of the sample represented in image 1a.

Figure 3. AFM generated images of $\mathbf{2} \mathbf{a} / \mathbf{b}$ dot patterns after backfilling the bare gold surface with ODT. (a) level topography demonstrated by tapping-mode AFM (b) frictional force variations revealing CTV-lipoate spots surrounded by ODT by LFM.

Figure 4. AFM images of the samples after $\mathrm{C}_{60}$ deposition. a) Tapping-mode AFM image showing a height increase of $\sim 1.0 \mathrm{~nm}$ is observed where the CTV-disulfide ink was patterned, but not to the surrounding ODT surface. b) The frictional contrast between CTV-C 60 is apparent relative to the backfilled ODT surface in the LFM image. c) Cross-sectional step height profile from tapping-mode AFM shows the periodic height increase of $\sim 1.0 \mathrm{~nm}$ on the sample.

Figure 5. Proposed $\mathrm{C}_{60}$ binding to the apex-modified, surface-bound CTV. 


\section{Figure 1}
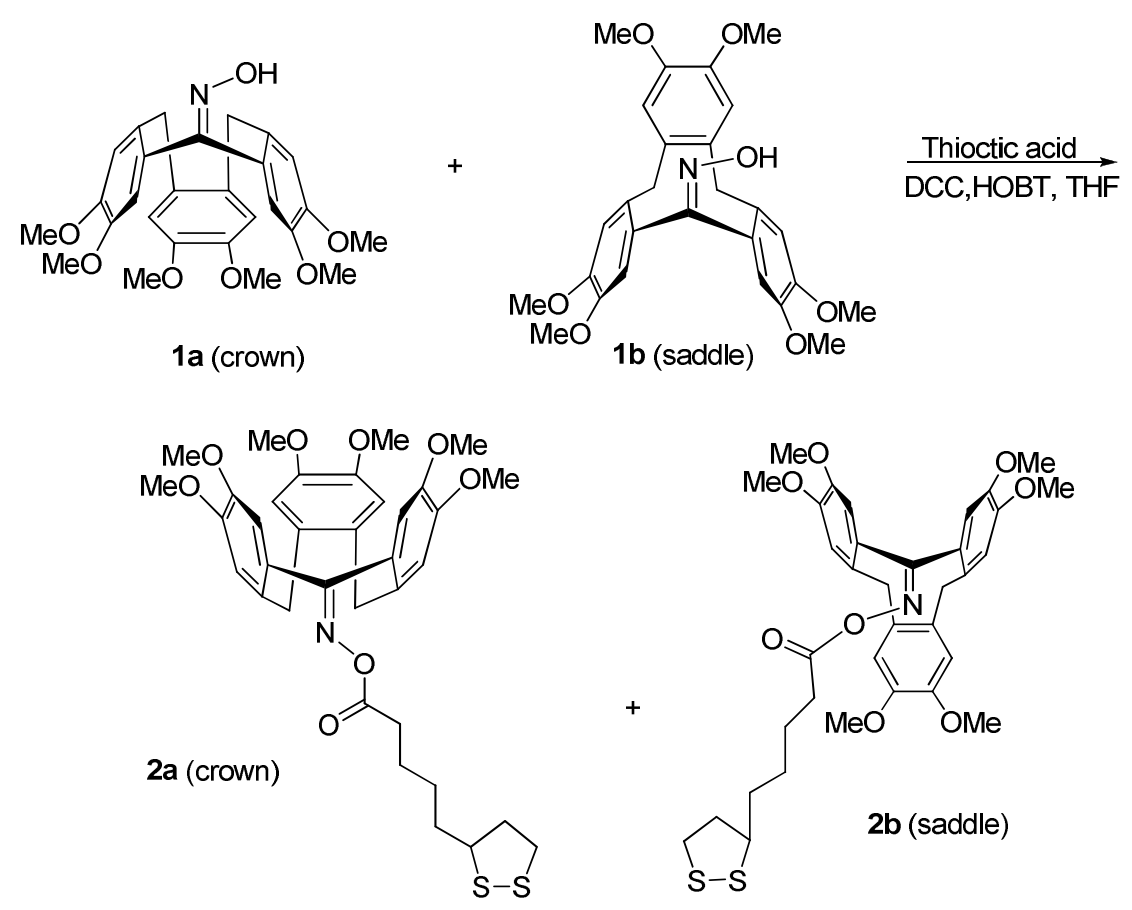
Figure 2
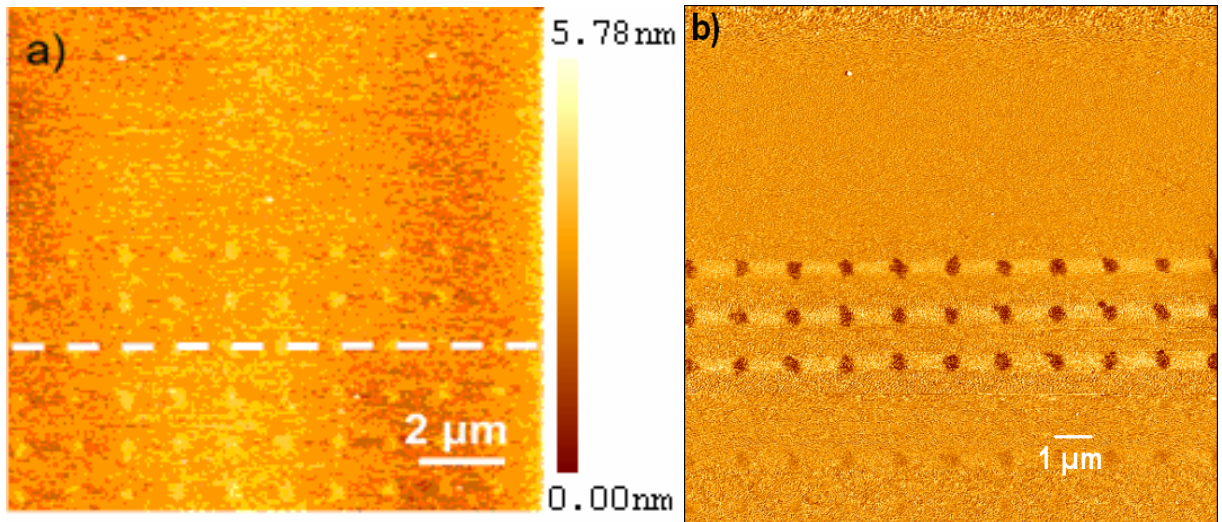

c)

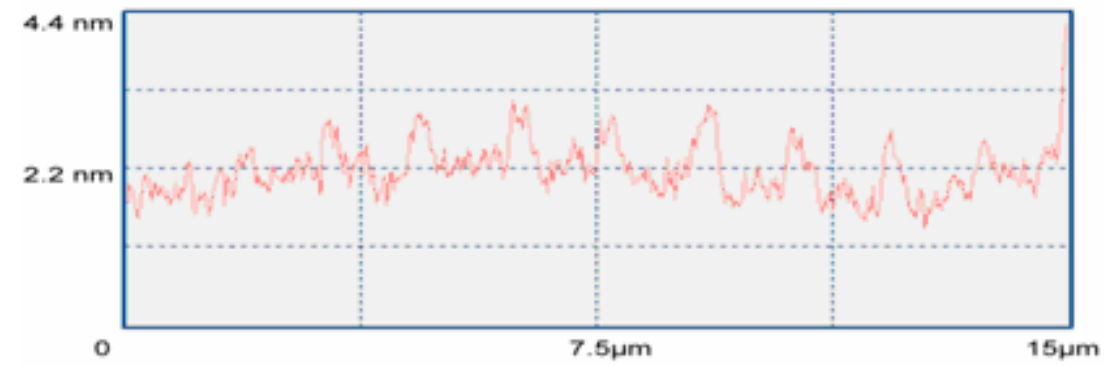




\section{Figure 3}
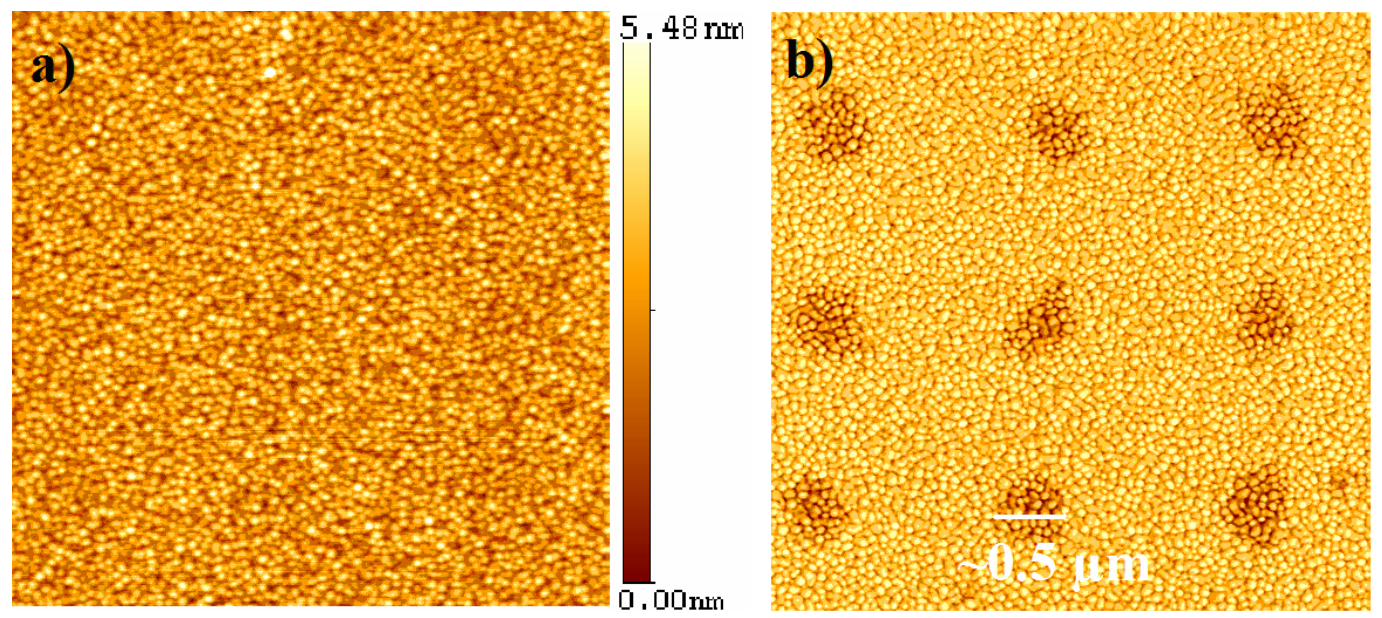

c)

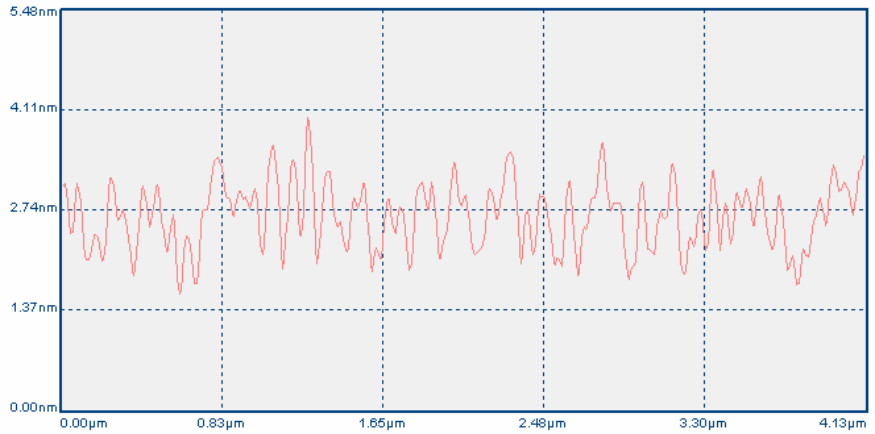


Figure 4
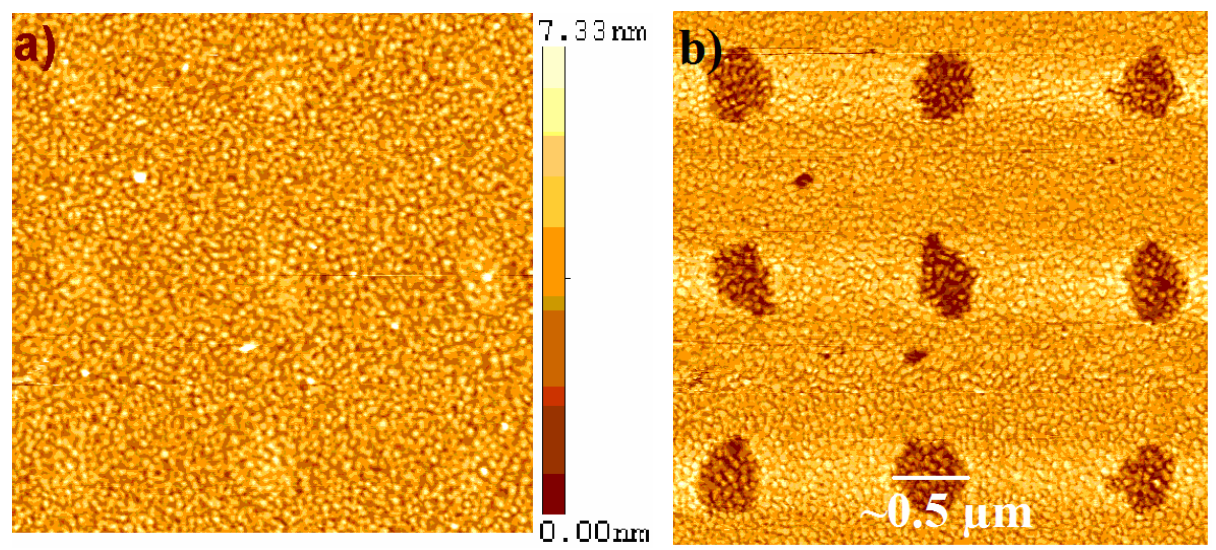

c)

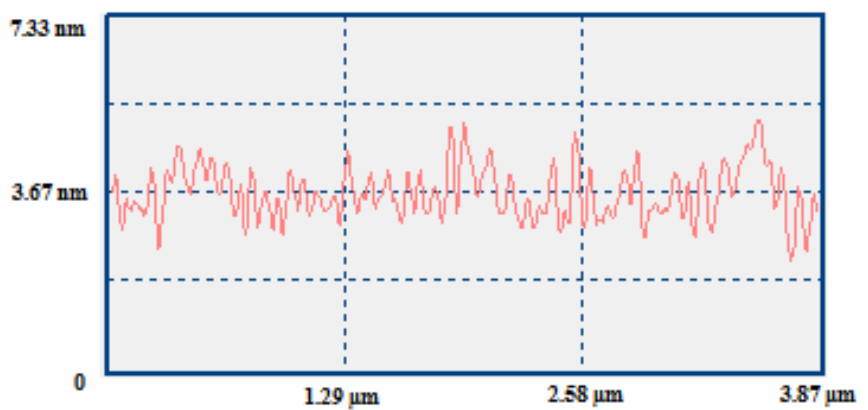


Figure 5

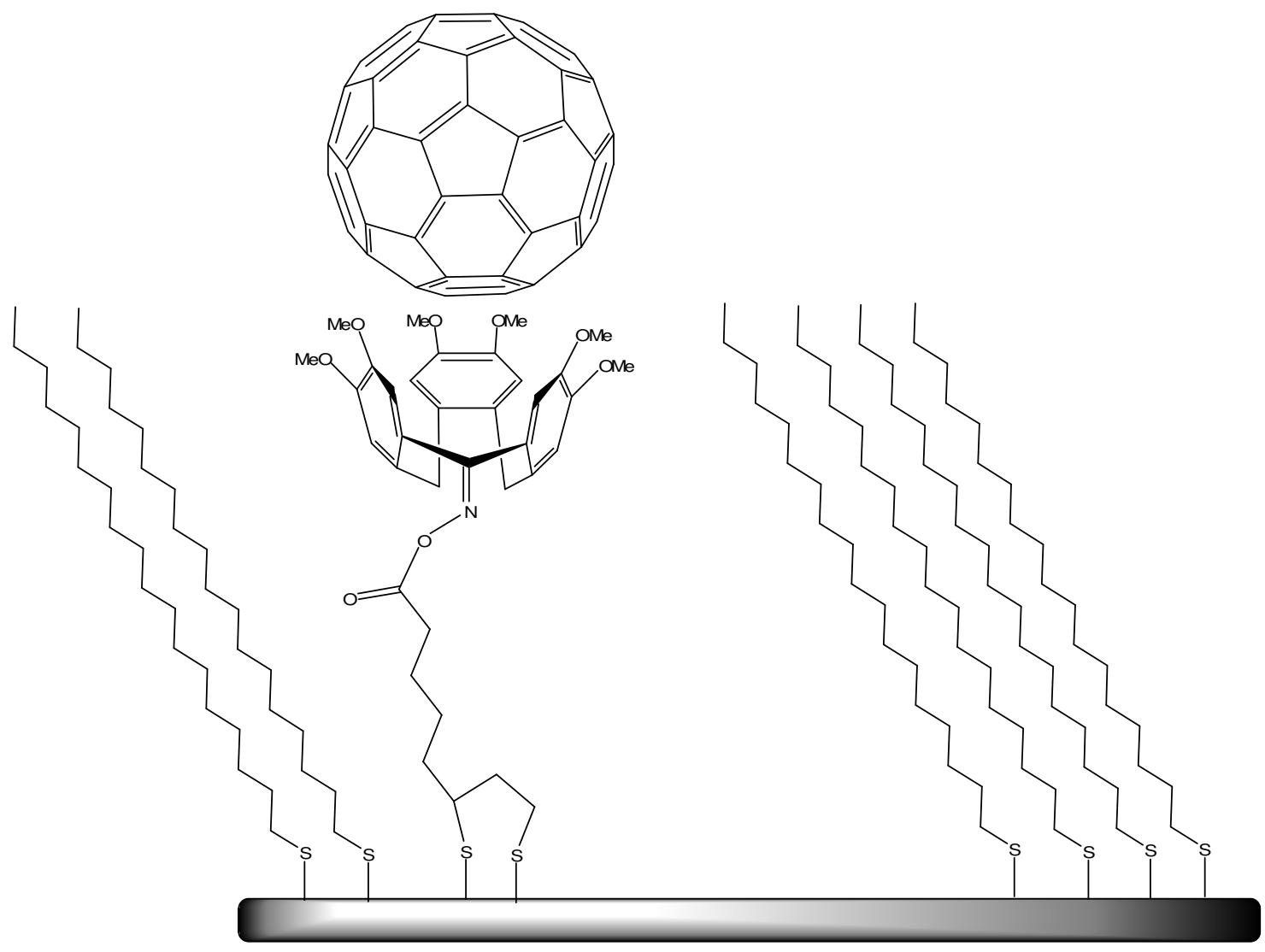

